\title{
An Introduction to Evolutionary Developmental Biology
}

\author{
Katherine E. Willmore
}

Published online: 8 June 2012

(C) Springer Science+Business Media, LLC 2012

What is evolutionary developmental biology? While the question is good, the answer remains somewhat unsatisfying. In the broadest sense, evolutionary developmental biology - or evo-devo-is the study of the interaction between developmental processes and evolutionary factors. However, this definition does not speak to the overarching aims of evo-devo, or to the type of research that falls under its umbrella. The reason for this vagueness stems from conflicting opinions on the aims and scope of evo-devo research. This conflict is well demonstrated by the many names that are often used synonymously with evolutionary developmental biology: evolution of development, developmental evolution, comparative development, and microevolution of development (Hall 2000a).

These differing monikers point to the fundamental aspect of the confusion in defining evo-devo. As a distinct field of biological study, is evo-devo defined by the set of tools used for its study, or by the set of questions it aims to answer? I agree with the many authors who have asserted that as an autonomous discipline, evo-devo is defined by the distinct evolutionary problems it seeks to solve (Atkinson 1992; Brigandt and Love 2010; Hall 2000a; Hendrikse et al. 2007; Love and Raff 2003; Love 2010).

If evo-devo is defined by its central question or problem, then what is this question? While there are conflicting views regarding the central concept of evo-devo, the answer proposed by Hendrikse and colleagues (2007) focuses on a concept that is unique to evo-devo and that embodies all current approaches to research within the discipline. As the

K. E. Willmore $(\bowtie)$

Department of Anthropology, Pennsylvania State University,

409 Carpenter Building, University Park,

State College, PA 16802, USA

e-mail: katwillmore@gmail.com title of their paper plainly suggests, evolvability — or the capacity of developmental systems to evolve - is the proper focus of evo-devo. They argue that developmental processes determine variation by influencing the amount of variation generated as well as biasing the direction of variation generated. Since selection acts on variation to produce evolutionary changes, understanding how variation is generated and modulated through developmental processes is the core of evo-devo. Therefore, following Hendrikse et al. (2007), I suggest that evo-devo is the study of how developmental processes generate and modulate variation.

Why is it necessary to have this subdiscipline of evolutionary biology? The traditional answer is that development was left out of the Modern Synthesis of evolutionary theory. Therefore, the majority of textbooks on evolution neglect the effects of development on evolutionary change (though this trend is rapidly changing). While this "new" synthesis was effective in describing how changes within and among species arise through population-level changes in gene frequency, it could not provide a satisfactory explanation for the origin of organismal form (Müller 2007). Adding a developmental component to evolutionary theory - in the manner of evo-devo - provides the conceptual and mechanistic tools necessary to search for such explanations.

Despite its relatively recent resurgence, the connection between development and evolution is not new. Darwin considered embryonic studies as essential to his theory of evolution, and long before Darwin published Origin, the connection between evolutionary biology and developmental biology (known then as embryology) was recognized. In fact, the term evolution that we now associate with phylogenetic change initially described ontogenetic (or developmental) change (Hall 1999).

Despite the rich historical connection between development and evolution, early in the twentieth century a sharp 
divergence arose in the way that researchers approached the study of evolutionary theory. These divergent approaches stemmed from practices used in embryology and the new field of genetics (Burian 2000). These two fields of study used different standards of evidence and model systems and looked at different sets of problems (Atkinson 1992; Burian 2000; Love 2009). A major reason for the divergence between embryology and genetics was the even earlier conceptual split that occurred within embryology. In the late nineteenth century, the study of embryology was divided into comparative embryology and experimental embryology (Hall 2000b; Love and Raff 2003). Comparative embryology was largely descriptive and was invested in evolutionary problems such as constructing phylogenies (Atkinson 1992; Love 2009). Experimental embryology broke away from comparative embryology, favoring an empirical approach to the study of development (Atkinson 1992; Love 2009). Additionally, the focus of experimental embryology moved away from evolutionary problems. By the turn of the twentieth century, work in comparative embryology was overshadowed by the largely popular upsurge in experimental biology. Therefore, while productive research in comparative embryology continued over the next century, it received little attention and was left out of the Modern Synthesis (Love and Raff 2003; Olsson et al. 2009, 2010).

Ironically, developmental genetics - a descendant of experimental embryology - is credited for the recent reunion of evolution and development (Arthur 2002; Carroll et al. 2005; Gilbert 2003; Müller 2007; Wilkins 2002). Indeed, the discovery made in the early 1980 s using developmental genetics that all animals share a set of genes known as Hox genes is largely responsible for dragging evo-devo out of the shadows. While the discovery of Hox genes and subsequent applications of developmental genetics to evolutionary problems have been profoundly successful and have popularized evo-devo as a discipline, we must remember that developmental genetics provides a tool for studying evodevo rather than defining it as a field of study (Love and Raff 2003). Pioneering works in the conceptual reunion of development with evolution are Gould's (1977) book Ontogeny and Phylogeny, Riedl's (1978) book Order in Living Organisms, and Jacob's (1977) paper on evolution by tinkering. These works focused on the conceptual themes embodied by evo-devo, such as heterochrony, developmental constraints, the origins of body plans and novelties, integration and modularity, phenotypic plasticity and the role of environmental influences on evolution, and robusticity in developmental systems. Evodevo arose as a distinct discipline in the latter third of the twentieth century from the fusion of these conceptual works and the application of tools from developmental genetics, molecular biology, and other disciplines such as geometric morphometrics.
Today, evo-devo is commonly described as having "arrived" as an autonomous, professionally recognized discipline (Atkinson 1992; Gilbert 2000; Hall, this issue; Hendrikse et al. 2007; Laubichler and Wagner 2004; Love and Raff 2003). Indeed, professionally, evo-devo has arrived with several academic journals that focus exclusively on the discipline, representation within professional societies, as well as academic positions, textbooks, classes, workshops, and research grant panels that are devoted to the field. However, I argue that evo-devo is underrepresented in discussions of evolutionary biology aimed at the nonscientist. The goal of this issue is to help make this exciting field of study accessible to a larger audience. The papers and essays in this issue introduce readers to many of the major themes of evo-devo research.

Brian Hall connects past discoveries with current efforts in evo-devo, and in so doing demonstrates the deep roots of what we now consider new and groundbreaking research. Having been a part of the resurgence of evo-devo from the beginning, he offers a well-informed projection of where the field is headed in terms of the sorts of tools that might be used and the questions that might be asked. Ken Weiss traces the development of an organism from the fertilized egg to the fully formed adult. His logical progression through development eases the reader through difficult concepts such as inheritance, interaction, and cooperation, concepts that are commonly misunderstood but are rarely clearly presented. Ken McNamara discusses heterochrony, a concept that dominated evo-devo when the field reemerged in the 1970s, which continues to be a major theme in current research. Using a wide variety of examples, he demonstrates how changes in the timing or rate of developmental events can influence evolutionary trends in morphological features and life history traits. My paper explores the origin and maintenance of the basic body plan. I attempt to show how this somewhat antiquated concept plays a central role in much of current evo-devo research. Evolvability is explored by Mihaela Pavlicev and Gunter Wagner. In their paper, they make the complex concept of evolvability accessible, a challenging feat. They also describe how this central concept is related to other major themes in evo-devo, namely modularity and robustness.

A common thread running through these papers is how the ideas presented are put into an historical context. This historical perspective demonstrates to those unfamiliar with evo-devo the depth of the ideas that are embodied by the discipline. While the popularity of evo-devo in professional circles is relatively recent, the ideas, questions, and concepts of the discipline have interested researchers since before Darwin introduced his theory of evolution. It is my hope that accessible presentations of evo-devo concepts, such as the papers in this special issue, will result in the same 
upsurge of interest in evo-devo for nonscientists as it has for professional biologists.

Acknowledgments I would like to extend a special thank you to all of the authors that contributed to this issue. I would also like to thank Niles, Michelle, and Greg Eldredge for the opportunity to assemble and contribute to this special issue. I greatly appreciate their support, guidance, and patience.

\section{References}

Arthur W. The emerging conceptual framework of evolutionary developmental biology. Nature. 2002;415:757-64.

Atkinson JW. Conceptual issues in the reunion of development and evolution. Synthese. 1992;91:93-110.

Brigandt I, Love AC. Evolutionary novelty and the evo-devo synthesis: field notes. Evol Biol. 2010;37:93-9.

Burian RM. General Introduction to the symposium on evolutionary developmental biology: paradigms, problems, and prospects. Am Zool. 2000;40:711-7.

Carroll SB, Grenier JK, Weatherbee SD. From DNA to diversity: molecular genetics and the evolution of animal design. 2nd ed. New York: Blackwell; 2005.

Gilbert SF. Diachronic biology meets evo-devo: C.H. Waddington's approach to evolutionary developmental biology. Am Zool. 2000;40:729-37.

Gilbert SF. The morphogenesis of evolutionary developmental biology. Int J Dev Biol. 2003;47:467-77.

Gould SJ. Ontogeny and phylogeny. Cambridge: Harvard University Press; 1977.
Hall BK. Evolutionary developmental biology. 2nd ed. Dordrecht: Kluwer; 1999.

Hall BK. Evo-devo or devo-evo: does it matter? Evol Dev. 2000a;2:177-8.

Hall BK. Balfour, Garstang and de Beer: the first century of evolutionary embryology. Am Zool. 2000b;40:718-28.

Hendrikse JL, Parsons TE, Hallgrímsson B. Evolvability as the proper focus of evolutionary developmental biology. Evol Dev. 2007;9:393-401.

Jacob F. Evolution and tinkering. Science. 1977;196:1161-6.

Laubichler MD, Wagner GP. Introduction to the papers of the 2001 Kowalevsky Medal winner symposium. J Exp Zool (Mol Dev Evol). 2004;302B:1-4.

Love AC. Marine invertebrates, model organisms, and the modern synthesis: epistemic values, evo-devo, and exclusion. Theory Biosci. 2009;128:19-42.

Love AC. Rethinking the structure of evolutionary theory for an extended synthesis. In: Müller G, Pigliucci M, editors. Evolution: the extended synthesis. Cambridge: MIT Press; 2010. p. 403-41.

Love AC, Raff RA. Knowing your ancestors: themes in the history of evo-devo. Evol Dev. 2003;5:327-30.

Müller GB. Evo-devo: extending the evolutionary synthesis. Nature. 2007;8:943-9.

Olsson L, Hoßfeld U, Breidbach O. Preface. Between Ernst Haeckel and the homeobox: the role of developmental biology in explaining evolution. Theory Biosci. 2009;128:1-5.

Olsson L, Levit GS, Hoßfeld U. Evolutionary developmental biology: its concepts and history with a focus on Russian and German Contributions. Naturwissenschaften. 2010;97:951-69.

Riedl R. Order in living organisms: a systems analysis of evolution. New York: Wiley; 1978.

Wilkins AS. The evolution of developmental pathways. Sunderland: Sinauer Associates; 2002. 